\title{
1 Electronic Health (E-Health) und Mobile Health (mHealth) - Ein Definitionsversuch
}

\author{
David Matusiewicz und Christian Thielscher
}

Der vorliegende Beitrag versucht, die beiden Begriffe Electronic Health (E-Health) und Mobile Health (mHealth) zu definieren. „E-Health“, „mHealth“ oder „M-Health“ lassen sich ebenso wie verwandte Begriffe - wie „Telemedizin“ oder "Gesundheitstelematik“ - nur schwer fassen, und zwar aus mehreren Gründen: Erstens handelt es sich nicht um physikalische Eigenschaften von etwas, sondern um Zuschreibungen, und zweitens verändert sich der Inhalt dieser Begriffe über die Zeit. So würden die meisten Autoren heute z.B. das einfache Telefongespräch zwischen einem Patienten und seinem Arzt auch dann nicht als „E-Health“ bezeichnen, wenn beide ein internetgestütztes Telefon verwenden. Der Begriff „E-Health“ impliziert daher immer auch, dass es sich um neue Technologien oder Anwendungen handelt; und diese Neuigkeit ist jeweils nur einige Zeit lang neu.

\section{E-Health}

Unter dem Begriff „Electronic Health“ (abgekürzt „E-Health“) werden elektronisch unterstützte Aktivitäten und Systeme im Gesundheitswesen zusammengefasst, die Patientendaten und andere medizinische Informationen über Entfernungen hinweg erheben, verfügbar machen und/oder auswerten und dabei Techniken einsetzen, die noch nicht als Standard empfunden werden. E-Health wird von vielen Autoren als ein Oberbegriff für die Gesamtheit aller solcher elektronischen Anwendungen zur medizinischen Versorgung verstanden. Eine definitorische Abgrenzung zur „Telemedizin“ ist schwierig; häufig werden die beiden Begriffe als Synonyme verwendet. 
E-Health erfolgt auf Basis von modernen Informations- und Kommunikationstechnologien (IKT). Diese IKT werden als Schlüsseltechnologien im Gesundheitswesen gesehen (siehe auch Thielscher 2007). So soll den Herausforderungen im Gesundheitssystem, die insbesondere durch den demografischen Wandel und die Versorgung in strukturschwachen und ländlichen Gebiete geprägt sind, u.a. mithilfe von flächendeckenden Anwendungen der Telematik und Telemedizin begegnet werden. Anwendungen von E-Health betreffen insbesondere Information, Kommunikation, Dokumentation und Vernetzung, z.B. in Form von Teleedukation/Teleausbildung, Telekonsultation, Telediagnostik, Telemonitoring und die Teletherapie.

Für die Entwicklung von E-Health spielen zahlreiche Disziplinen eine Rolle. Hierzu zählen u.a. die Betriebs- und Volkswirtschaftslehre, Kommunikations- und Medienwissenschaften, medizinische Informatik, Versorgungsforschung und - selbstverständlich - die Medizin. Heute gibt es zahlreiche Fachgesellschaften, die sowohl auf der nationalen als auch internationalen Ebene agieren und die Thematik rund um E-Health vorantreiben.

Aktuell wird versucht, in Deutschland die Vernetzung aller Institutionen und Personen im Gesundheitswesen auf Basis einer sicheren und verlässlichen Plattform, der Telematikinfrastruktur, zu ermöglichen.

\section{Entwicklung des Begriffes}

Der Begriff hat sich in den letzten Jahren auch durch die Zunahme der technischen Möglichkeiten und Anwendungsgebiete weiterentwickelt. In den 1960erJahren wurde u.a. von Teleradiologie gesprochen, in den 1970er-Jahren von Telemedizin, in den 1990er-Jahren von Telematik und in der aktuellen New Economy wird gern der Oberbegriff E-Health verwendet. Der Begriff wird oftmals synonym verwendet mit den Begriffen, die durch die Begriffsentwicklung entstanden sind, und auch dem Begriff Health 2.0.

Als Schlüssel zur Telematikinfrastruktur wird die elektronische Gesundheitskarte (eGK) gesehen, die die alte Krankenversicherungskarte (KVK) ersetzt hat. Ursprünglich war ihre Einführung zum 1. Januar 2006 vorgesehen; dieses Ziel wurde u.a. durch ungeschickte Vorgehensweise (z.B. Verpflichtung für alle Patienten, unklarer Datenzugang) um Jahre verfehlt. Seit dem 1. Januar 2015 gilt die eGK nunmehr als ausschließlicher Berechtigungsnachweis für die Inanspruchnahme von Leistungen in der gesetzlichen Krankenversicherung (GKV). Mit der eGK sollen auch die Qualität der medizinischen Versorgung verbessert und die Informationsmöglichkeiten der Patienten gestärkt werden.

In Deutschland gibt es nach wie vor Probleme hinsichtlich der Nutzung von E-HealthSystemen. Bereits vorhandene EDV-Systeme sind bislang häufig nicht vernetzt; vielfach handelt es sich um Insellösungen - also Teillösungen, die nicht miteinander kommunizieren und auch nur schwer interoperabel gemacht werden können. Auch sind die immer wieder auftretenden Datenlecks und andere, gravierende Sicherheitslücken gängiger Computer- bzw. Softwaresysteme (zuletzt z.B. vom Virus „wanna cry“ offen gelegt) in der Medizin schlicht inakzeptabel. Daher verlangen z.B. die kassenärztlichen Vereinigungen aus Datenschutzgründen sogenannte Stand-AloneRechner. Auch die daraus resultierenden Diskussionen um die Ausgestaltung (und 
Vergütung) telemedizinischer Leistungen und den Datenschutz tragen dazu bei, dass die Weiterentwicklung von E-Health in Deutschland nur schleppend vorangeht.

Das Bundesministerium für Gesundheit (BMG) hat ein „Gesetz für sichere digitale Kommunikation und Anwendungen im Gesundheitswesen (E-Health-Gesetz)“ erarbeitet, welches zum 1. Januar 2016 in Kraft getreten ist. Das Gesetz enthält einen Fahrplan für die Einführung einer digitalen Infrastruktur mit hohen Sicherheitsstandards und die Einführung nutzbringender Anwendungen auf der elektronischen Gesundheitskarte. Ziel des Gesetzes ist es, die Digitalisierung der Gesundheitsversorgung voranzutreiben und einen Rechtsrahmen für die digitale gesundheitsbezogene Kommunikation zu setzen. Die einzelnen Akteure der Selbstverwaltung erhalten hierbei klare Vorgaben und Fristen, die bei Nichteinhaltung zu Sanktionen führen werden. Die inhaltlichen Schwerpunkte des Gesetzentwurfes sind u.a. die Einführung und Nutzung medizinischer Anwendungen (elektronischer Arzt- und Entlassbrief, einheitliche Medikationspläne, Notfalldaten), der Ausbau der Telematikinfrastruktur und die Förderung telemedizinischer Leistungen. Auch soll die Struktur der Gesellschaft für Telematikanwendungen der Gesundheitskarte mbH (gematik), die für die Einführung der eGK verantwortlich ist, verbessert werden. Insgesamt steht das Gesetz für eine telemedizinisch und mobil ausgerichtete Digitalisierung des Gesundheitswesens und sorgt derzeit für Diskussion unter Befürwortern und Kritikern. Mit derzeit mehr als sechs Millionen Beschäftigten im Gesundheitswesen bekommt das Thema E-Health insgesamt eine wachsende Bedeutung; durch die zunehmende Spezialisierung auch neue Berufsfelder entstehen werden.

\section{mHealth}

Als „Mobile Health“ (abgekürzt „mHealth“) wird eine Untergruppe von E-Health-Aktivitäten und -Systemen bezeichnet, die auf mobilen Geräten angeboten werden. Hierbei erfolgt der Einsatz von Mobilfunktechniken und mobilen und drahtlosen Technologien.

Merkmale von mHealth beziehen sich einerseits auf die technischen Voraussetzungen wie mobile Computer, Smartphones sowie andere Endgeräte, andererseits auf die damit verbundenen Kommunikationsmethoden.

Einsatzgebiete von mHealth umfassen alle Versorgungsbereiche, also Prävention, Diagnostik, Therapie, Nachsorge, Monitoring von Patienten und bei manchen Autoren auch Wellnessthemen. Außerdem werden mHealth-Lösungen auch im administrativen Bereich z.B. zur Unterstützung des Praxis- oder Klinikmanagements verwendet. Ziel von mHealth ist die Verbesserung der medizinischen Versorgung durch die Nutzung von mobilen Technologien. Dies gilt insbesondere für ländliche Regionen. Zusätzlich sollen die Anwendungen dazu beitragen, einen verantwortungsvollen Umgang mit der eigenen Gesundheit zu erreichen und durch die Bereitstellung von Gesundheitsinformationen die Gesundheitskompetenz der Patienten zu erhöhen. 
Die Anwendung von mHealth berührt grundlegende Sicherheitsansprüche v.a. der Patienten; daher sind die Datenschutzbeauftragten des Bundes und der Länder ebenso wie die übrigen Akteure im Gesundheitswesen gefordert.

Die zunehmende Verbreitung des Internets und die zunehmende Nutzung von Smartphones und Tablets führen dazu, dass mHealth für Krankenkassen, Leistungserbringer und auch Patienten bzw. Versicherte wichtiger wird. Als treibende Kraft dieser Innovation gelten insbesondere Start-up-Unternehmen; die Kostenträger (Krankenkassen) und andere Körperschaften reagieren - auch aufgrund der bisher nicht geklärten Rechtslage - eher zurückhaltend. Das Marktpotenzial von mHealth in Deutschland ist noch schwerer zu beziffern als das von E-Health. Aufgrund der Tatsache, dass Gesundheits-,„Apps“ überwiegend nicht von den privaten und gesetzlichen Krankenkassen vergütet werden, sind sie insbesondere für den sogenannten zweiten Gesundheitsmarkt interessant.

Eine wichtige aktuelle gesundheitspolitische Diskussion ist daher, inwieweit die Einbindung von mHealth in das Vergütungssystem der gesetzlichen und privaten Krankenkassen erfolgen kann, und inwiefern die medizinischen Leistungserbringer und Patienten diese Entwicklung weiter vorantreiben werden.

\section{Literatur}

Swart E, Ihle P, Gothe H, Matusiewicz D (2014) Routinedaten im Gesundheitswesen - Handbuch Sekundärdatenanalyse. Grundlagen, Methoden und Perspektiven, 2. Auflage, Bern

Thielscher C (2007) Zukunft der Telemedizin in Deutschland. In: Anycare (Hrsg.) Telemedizin - Innovationen für ein effizientes Gesundheitssystem. Thieme, Stuttgart 